Relations industrielles

Industrial Relations

\title{
Contemporary Collective Bargaining, by Harold W. Davey, New Jersey, Prentice-Hall Inc., Englewood Cliffs, 1972, 395 pp.
}

\section{Jean Bernier}

Volume 28, numéro 3, 1973

URI : https://id.erudit.org/iderudit/028430ar

DOI : https://doi.org/10.7202/028430ar

Aller au sommaire du numéro

Éditeur(s)

Département des relations industrielles de l'Université Laval

ISSN

0034-379X (imprimé)

1703-8138 (numérique)

Découvrir la revue

Citer ce compte rendu

Bernier, J. (1973). Compte rendu de [Contemporary Collective Bargaining, by Harold W. Davey, New Jersey, Prentice-Hall Inc., Englewood Cliffs, 1972, 395 pp.] Relations industrielles / Industrial Relations, 28(3), 668-669.

https://doi.org/10.7202/028430ar

Tous droits réservés (C) Département des relations industrielles de l'Université Laval, 1973
Ce document est protégé par la loi sur le droit d'auteur. L'utilisation des services d'Érudit (y compris la reproduction) est assujettie à sa politique d'utilisation que vous pouvez consulter en ligne.

https://apropos.erudit.org/fr/usagers/politique-dutilisation/ 
train de fournir la meilleure preuve de cette affirmation.

Enfin, et cette dernière remarque s'applique surtout au Québec, l'auteur avance que l'on a inscrit la négociation et les règles pour la diriger dans un contexte d'entreprise privée où la force d'impulsion dans les conditions de travail proviendrait et de la grande entreprise et d'un syndicalisme qui, parce que supposément 《d'affaires》, respecterait cette règle du jeu. Encore une fois cette affirmation est corroborée par la récente politisation du conflit mettant aux prises le gouvernement du Québec et le Front commun intersyndical.

Le dernier article qui sert également de conclusion à ce séminaire est celui de John Crispo, directeur du Centre de relations industrielles de l'Université de Toronto. Comme Me Lapointe, le professeur Crispo rejette le système d'arbitrage obligatoire institutionnalisé comme mécanisme de règlement des conflits d'intérêts dans le secteur public. Sa position est que l'on doit accorder (ou maintenir) le droit de grève, sauf dans les cas où les services essentiels sont menacés, car l'expérience canadienne révèle que notre société à date s'est fort bien accommodée des grèves dans le secteur public. Le professeur Crispo n'est pas très explicite quant aux procédures à suivre dans les cas où les services essentiels sont menacés, se contentant d'affirmer que la législation ne devrait rien prévoir de précis afin que les parties soient dans le doute quant au type d'intervention éventuelle. Cette solution nous semble trop facile, car elle escamotte le cas très important où le gouvernement provincial est en même temps l'employeur et celui qui doit décider de la forme d'intervention à utiliser.

La conclusion du professeur Crispo est très optimiste : en comparant la situation actuelle du secteur public avec celle du secteur privé il y a trente ans alors que le Canada et les Etats-Unis étaient affligés de nombreuses grèves, Celui-ci conclut qu'une période de stabilisation identique à celle qui a suivi ces années turbulentes dans le secteur privé, pourrait fort bien succéder au chaos actuel qui existe dans le secteur public.

\section{Université Laval}

Contemporary Collective Bargaining, by Harold W. Davey, New Jersey, Prentice-Hall Inc., Englewood Cliffs, 1972, $395 \mathrm{pp}$.

Ce livre constitue la troisième édition de cet ouvrage, la précédente datant déjà de 1959. Compte tenu du caractère dynamique de la négociation collective et de l'évolution constante du contenu des conventions qui en résultent, l'auteur n'a pas voulu se limiter à de simples retouches en vue d'atteindre à une mise-à-jour de son livre. En fait, l'édition qu'il nous présente maintenant constitue dans une large mesure une refonte complète de la précédente.

Sans avoir abandonné les thèmes traditionnels de la convention collective tels que les droits de la direction, le règlement des griefs, l'arbitrage et l'ancienneté, au sujet desquels il a parfois repris textuellement des extraits de certains chapitres de l'édition de 1959, l'auteur aborde dans le présent volume des sujets qui ont acquis ces dernières années une place importante dans la négociation. C'est ainsi par exemple qu'il accorde beaucoup plus d'importance qu'en 1959 aux problèmes relatifs à la sécurité d'emploi et aux bénéfices marginaux. Un chapitre entièrement nouveau est consacré à la négociation collective par les employés du gouvernement et par certaines catégories de professionnels tels que les enseignants et les infirmières.

En dépit de ces refontes et de ces additions, la perspective globale de l'ouvrage demeure sensiblement la même que dans l'édition précédente en ce sens que l'auteur paraît avoir tenté de maintenir le difficile juste milieu entre l'exposé purement théorique et l'analyse appliquée à des cas pratiques au risque même de décevoir quelque peu les uns et les autres, théoriciens et praticiens. Néanmoins les premiers y trouveront leur profit grâce aux abondantes références d'ordre bibliographique rapportées à la fin de chaque chapitre, et les seconds auront l'occasion de se familiariser avec une multitude de problèmes que pose la négociation collective au niveau de l'entreprise ou de l'établissement.

A cet égard, il s'agit d'un ouvrage dont il ne faut pas tant attendre une explication scientifique des phénomènes 
reliés à la négociation collective mais bien davantage une sorte d'état de la situation dont l'exposé est riche de l'expérience de l'auteur dans les domaines de l'enseignement et de l'arbitrage qui fut l'occasion pour lui d'entrer en contact avec les problèmes concrets qui résultent de la négociation et de l'application des conventions collectives. Cette expérience transparait tout au long de l'ouvrage aussi bien au niveau de l'illustration des problèmes abordés que dans l'approche analytique elle-même.

Dans cette perspective, cet ouvrage peut être considéré comme un très bon livre d'introduction à la négociation collective aussi bien pour l'étudiant que pour le praticien.

\section{Jean BERNIER}

\section{Université Laval}

\section{Collective Bargaining and the Challenge} of New Technology, Geneva, International Labour Office, 1972, $71 \mathrm{pp}$.

En 1963, l'Organisation internationale du travail adoptait une recommandation (no 119) concernant la cessation de la relation de travail à l'initiative de l'employeur. Cette recommandation non modifiée depuis son adoption, s'applique tant aux licenciements individuels que collectifs. Elle accepte entre autres les principes du licenciement pour motif valable, du préavis, du droit de recours des travailleurs, de l'utilisation des services publics et de la consultation. L'OIT propose que ces principes soient mis en application par une série de moyens dont la liste n'est sûrement pas exhaustive : loi, décrets, convention collective, décision arbitrale, etc.

Par cet ouvrage l'OIT veut faire un tour d'horizon des moyens prévus par certaines conventions collectives en certains pays pour appliquer les principes énoncés dans la recommandation no 119. $\mathrm{La}$ recherche que nous complétons présentement fait pour sa part un tour d'horizon des programmes publics directs visant l'application de ces mêmes principes aux Etats-Unis, en Grande-Bretagne, en France et au Québec.

Le sujet traité dans Collective Bargaining and the Challenge of New
Technology n'est sûrement pas nouveau. Ce qui fait le mérite particulier de cette publication est son caractère international et comparatif. C'est en fait un ouvrage du type de celui de Yves Delamotte, Les partenaires sociaux face aux problèmes de productivité et d'emploi, publié à l'OCDE. Cet aspect comparatif est sûrement très instructif, même pour le lecteur averti. Comme les auteurs le mentionnent au début de leur étude,

« Clearly, the bargaining context in each country is affected by different historical, political, economic, and cultural developments : the resulting collective agreements will therefore differ accordingly and demonstrate a variety of possible responses to the challenges of technological change ».

De plus, les auteurs se concentrent exclusivement sur ces conventions collectives qui indiquent clairement une réponse à l'introduction d'un changement technologique. Une telle contrainte nous empêche de connaître dans quelle proportion les parties s'attaquent réellement au problème des changements technologiques. En plus, on n'y retrouvera généralement pas de référence à ces clauses visant d'autres changements que les changements technologiques, ni à ces clauses ne visant qu'indirectement les changements technologiques.

Cette publication de l'OIT comprend deux chapitres. Le premier s'attarde aux différentes clauses directes adoptées en différents pays pour faire face aux problèmes posés par les changements technologiques. Le chapitre II, pour sa part, présente quelques accords d'entreprises ou d'industries au Canada. en France, en Allemagne et aux Etats-Unis. Il serait trop long ici de présenter en détail ces clauses ou ces accords décrits dans cette publication.

Même si, par la nature même du sujet traité, il ne faut sûrement pas s'attendre à une étude exhaustive, l'absence d'une conclusion générale représente une faiblesse sérieuse à cette publication, le sujet s'y prêtant très bien et l'expérience des auteurs le permettant sûrement. Le lecteur intéressé pourra cependant tirer ses propres conclusions à partir de cet ouvrage bien structuré et bien présenté. 